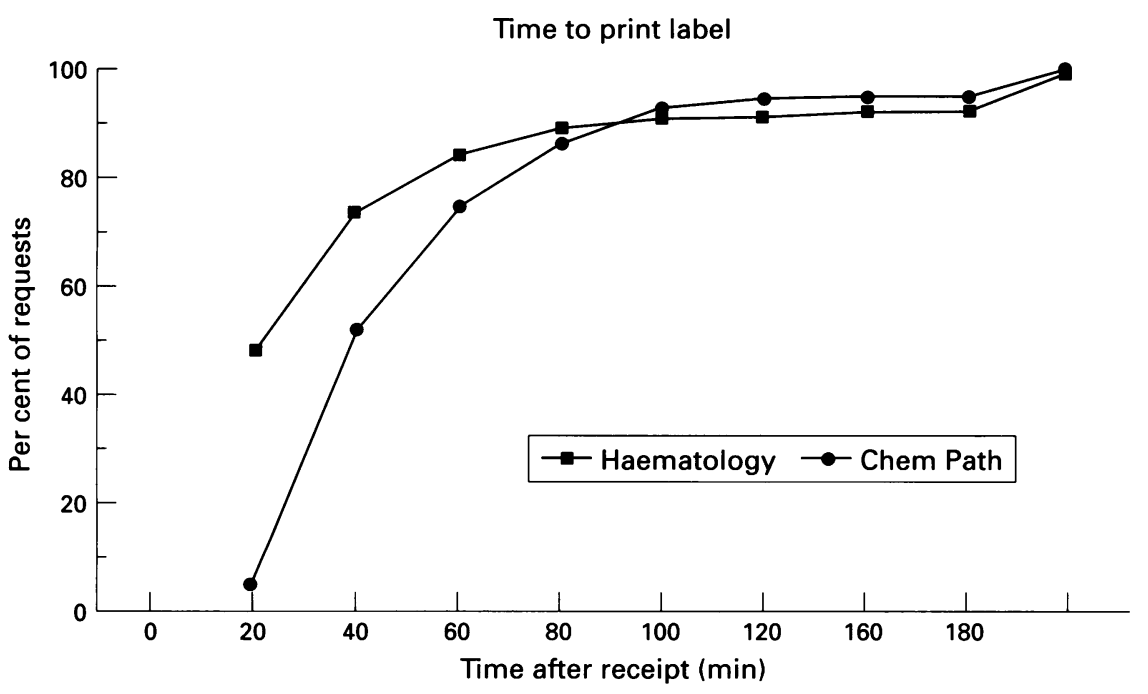

Figure 2 Time course of results processing providing hard copy of results as soon as they are available.

M J CLANCY

Department of Accident $\mathcal{E}$ Emergency Medicine

D BINGHAM

Department of Chemical Pathology Bristol Royal Infirmary

\section{Paracetamol overdose}

EDITOR - In relation to the short report on paracetamol overdose by Hulbert et al (volume 12, p 66), Evans Medical are currently varying the licence for Parvolex in order to make the labelling consistent with current treatment guidelines.

The product licence variation has to be submitted to and approved by the MCA Evans Medical are committed to updating the labelling as soon as possible and meanwhile would agree with the authors that the new guidelines of the UK Toxicology Group should be used in A\&E departments.

FRANCIS UPCHURCH
Medical Director
Evans Medical, Evans House,
Kingston Rd, Leatherhead,
Surrey KT22 $7 P Q$

\section{Anaphylactic shock}

EDITOR - Dr Brown must be commended on the high quality review article on anaphylactic shock. ${ }^{1}$ The erudite and comprehensive discussion of the "adrenaline dilemma" is particularly welcome.

In considering the administration of fluids, while colloid may well restore the circulation more effectively than crystalloid, Dr Brown neglects to mention that the colloid solutions themselves are proven to cause anaphylaxis from a histamine releasing effect. ${ }^{2}$ As many patients react to more than one antigen, it may be safer to use crystalloids so as to minimise the chance of adding fuel to the fire.
I would urge emergency physicians to educate their general practice colleagues, as well as their patients, in anaphylaxis, because in the community it is an infrequent but intimidating problem

In May 1994, the Chief Medical Officer urged that all patients suffering from peanut allergy should be referred to a specialis clinic. $^{3}$ It seems almost intuitive that good practice would require similar referral of all patients presenting with anaphylactic reactions, whatever their aetiology. The initiation of a supply of adrenaline and instruction in its use may be better supervised effect profile and the continuing uncertainty of dose and route; thereafter it can be monitored and rechecked by the general practitioner. As Epipen is still only available in the United Kingdom on a named patien basis, GPs can obtain only limited (if any) experience in its use. Adrenaline inhalers (Medihaler-Epi) give some relief, especially early in the attack, but again patients need training. Emergency physicians or immunologists will be more familiar with these preparations.

DAMIAN MCHUGH Accident E Emergency Medicin West Cumberland Hospital, Hensingham Whitehaven, Cumbria, UK

1 Brown AFT. Anaphylactic shock: mechanism and treatment. 7 Accid Emerg Med 1995; and treatm

2 Venboet $\mathrm{D}$ et al. Anaphylactic reactions to modified fluid gelatins. $\mathcal{f}$ Allergy Clin Immuno 1983;71:535-40.

3 CMO's Update 2. May 1994. London: De-

\section{The Patient's Charter}

EDITOR - I was interested in the short report by Cugnoni et al. ${ }^{1}$ In 1993 we carried out a related survey at Watford General Hospital (a suburban as opposed to inner city location). In addition to immediate assessment in accident and emergency (A\&E) we evaluated the National Charter standard of waiting time for an emergency ambulance and the early on by an immunologist, given its side partment of Health, 1994 patient's opinions as to the impact of the charter.

Over a six week period, 187 questionnaires were completed by $A \& E$ attenders, indicating that $62 \%$ were aware of the Patient's Charter. Eighty six per cent of these were able to define it correctly. Sixty six percent of responders recognised that a standard had been set regarding the waiting time for immediate assessment, of whom 44\% acknowledged that it had been quantified and $47 \%$ correctly replied that when seen, it would be by a nurse and so that their need for treatment could be assessed (rather than by any other member of the department or any further examination or treatment occurring)

Seventy five per cent of responders perceived that the charter had also set a standard for the waiting time for an emergency ambulance, $34 \%$ of these appreciating that it was 14 minutes (in an urban area). The majority were unable to quantify it.

With respect to the respondents' views of the effects of the charter on the emergency services, $46 \%$ believed that the initial waiting time in $A \& E$ had been altered $-64 \%$ though for the better. Thirty four per cent considered that the charter had affected the time they waited for an emergency ambulance; of these, $46 \%$ thought the waiting time had been reduced.

Overall $18 \%$ felt that the charter had been beneficial and $62 \%$ approved of the concept. We found a greater awareness of the charter and its specific standards than the study carried out at St Bartholomew's Hospital. However, we support the conclusions of Cugnoni $e t$ al, as we believe that our results probably also fall short of the hopes and expectations of both the patient and the NHS management executive.

MARIANNE JACKSON Newham General Hospital London E13, UK

1 Cugnoni HL, Hombrey PJH, Miles SAD. The Patient's Charter: views of patients attending an inner-city accident and emergency department. If Accid Emerg Med 1994;11: 264-5.

\section{Chest drain insertion}

EDITOR - We write with regard to the recent paper from Hassan and Keaney in which they cite winging of the scapula as an unusual complication of chest drain insertion. ${ }^{1}$ The Advanced Trauma Life Support system teaches "do no further harm" and that the safe way to insert a chest drain is to make an incision anterior to the midaxillary line rather than directly over it. ${ }^{2} \mathrm{Had}$ this protocol been followed the "complication" may have been avoided. FIONA POYNER Department of Accident and Emergency Queen's Medical Centre University Hospital NHS Trust Nottingham, UK

1 Hassan WU, Keaney NP. Winging of the scapula: an unusual complication of ches tube placement. $\mathcal{f}$ Accid Emerg Med 1995 12:156-7.

2 Advanced Trauma Life Support. Course for Physicians, 1993:138 